Giovanni Zorzi*

\title{
Captain Fantastic
}

di Matt Ross, U.S.A., 2016

Viggo Mortensen, dismessi da tempo i panni di Aragon nella trasposizione della trilogia del Signore degli Anelli che lo hanno reso celebre al pubblico mondiale e che gli hanno permesso di ritagliarsi uno spazio di diritto nella storia del cinema, collabora attivamente insieme al regista/sceneggiatore Matt Ross alla costruzione di un personaggio prezioso: un padre, Ben. L'attore statunitense, in uno stato di grazia che perdura ormai da anni (vedi il trittico con il regista David Cronenberg per il quale ha indossato anche i panni di Sigmund Freud), ci regala la possibilità di osservare e partecipare alle ambiguità di una figura genitoriale che dona ai propri figli l'incalcolabile privilegio di una profonda ed esperita educazione, confinandoli al tempo stesso ai margini di una società che sta per chiedere la loro partecipazione.

Ben ha scelto di crescere i suoi figli lontano dalla società consumistica, nel cuore di una foresta. In questo non-luogo i ragazzi si allenano fisicamente e intellettualmente: cacciano per procurarsi il cibo, studiano le scienze, i capolavori della letteratura e si confrontano in dibattiti democratici. La morte della madre, personaggio assente/presente, li costringerà a intraprendere un difficile viaggio nel mondo 'reale'.

Mortensen giganteggia affiancato dal cast riuscitissimo dei sei giovani attori-figli, che dall'infanzia alla tardo-adolescenza sono rappresentati come individui che vantano competenze da atleti, intellettuali e conoscitori critici di sistemi complessi (politica, economia, fisica...), ma che al tempo stesso ignorano saperi comuni della società odierna. Il film ci permette, grazie al racconto del loro primo vero viaggio nella società americana, di osservare l'incontro di due mondi che si scoprono come significativamente distanti ma ugualmente caratterizzati da limiti e ambivalenze. 
L'intimità costruita grazie anche all'approfondimento caratteriale dei protagonisti stimola una sorta di reciproca osservazione tra personaggi e spettatore. La nostra posizione non sembra infatti essere del tutto passiva durante la visione; osservando meravigliati il quotidiano di questa famiglia, ci sentiamo osservati come esseri extra-ordinari attraverso gli occhi dei figli alla scoperta della società consumista. L'ironia con cui veniamo scrutati costruisce brillanti sequenze, amaramente dissacranti il nostro concetto di ricchezza e benessere.

Il desiderio di conoscenza dei giovani protagonisti, certamente invogliato dalle stimolazioni paterne ad un sapere critico e curioso delle cose, diventa quindi parte di un percorso di incontro con l'altro e con le sue complessità. Tale incontro/scontro viene trattato anche attraverso l'ironia della commedia degli equivoci, costruita nel film attraverso alcuni misunderstanding che emergono nel dialogo con l'altro: 'Nike' per Nai è la dea della vittoria, per i suoi cugini una marca di scarpe; 'Doctor Spock' è per Bodevan il pediatra autore de Il bambino. Come si cura e come si alleva, per Claire il personaggio della serie televisiva Star Trek.

Altri momenti memorabili della rappresentazione del gruppo famiglia vengono riprodotti attraverso la messa in scena dello stile comunicativo intrafamiliare. Ben non usa giri di parole con i suoi figli, soprattutto quando deve affrontare argomenti di estrema sensibilità come il sesso, la malattia mentale o la morte. Tutti i suoi figli sono parimenti esposti alle sue sincere e dettagliate spiegazioni o risposte, senza una distinzione che renda ragione delle diverse fasi evolutive o del differente stato d'animo. Nei momenti meglio riusciti, siamo messi di fronte allo stress dei nostri taboo e della nostra morale, ridendone e al tempo stesso subendo una solleticazione del nostro fantasmatico, ritrovandoci sia schierati con la sincerità di Ben, che consapevoli della difficile gestione di questo approccio rigido e radicale. Tale antitesi vuole essere costruita nella messa in scena che confronta due padri, da una parte Ben, per il quale sembra non sussistere il non-detto, dall'altra suo cognato che tenta confusamente di difendere i propri figli dalla rappresentazione della drammatica morte della zia.

Stiamo parlando di una pellicola girata tra lo stato di Washington e il New Mexico, con gli stilemi del road-movie da e verso stati di frontiera, per un racconto il cui topos sembra essere proprio 'il limite'. C'è una narrazione tipica del film di confine, non solo geografico, che parte dalle foreste del nord-ovest e arriva ai deserti cari a Sergio Leone per descrivere i limiti dell'uomo Ben, padre che ha deciso di crescere i propri figli e costruire assieme alla moglie una società utopica che tuttavia, in quello che scopriamo essere un progressivo e collettivo percorso di formazione, incontra la finitezza della propria onnipotenza.

La struttura della società/famiglia/tribù affascina e sembra resistere (concettualmente, politicamente, affettivamente, economicamente...) 
grazie alla solida presenza dell'adulto/padre/capo. Ma cosa accade quando i confini non possono essere tratteggiati da Ben?

Alla crescita del sistema famiglia contribuisce il preziosissimo pensiero divergente dell'adolescenza. Se ne fa carico il figlio Rellian, che lo introduce già agli inizi del film in una scena di musica diegetica, dove l'intimo raccoglimento della famiglia attorno al fuoco, prima sottoforma di studio individuale e poi di collettiva interpretazione di una delicata melodia, viene scosso dalla sua forzata richiesta di un cambio di ritmo; armato di cajon e di uno sguardo che accenna la sua prossima sfida al pensiero e alle regole padre.

Lo sviluppo narrativo da romanzo di formazione sembra trovare il suo incipit nella suggestiva scena del rito di passaggio all'inizio del film, costruendo la prima antitesi, in questo caso sul concetto di adultità. Bodevan, figlio maggiore, è diventato uomo all'intero dell'utopia, ma lo vediamo disorientato nel mondo esterno, dove non ha ancora appreso le competenze per parlare con i/le pari. Il primo bacio di Bodevan con una ragazza allo spazio di sosta camper è un totale sconvolgimento e viene drammatizzato fino al riuscito anticlimax, strategia narrativa che ci permette di comprendere l'eccessiva distanza che i ragazzi vivono rispetto al mondo che stanno attraversando.

Possono i confini della mente e della famiglia essere quelli dello stare in società? Il film si e ci interroga su questo quesito che possiamo ulteriormente allargare a 'Può una grande conoscenza sopperire alla limitazione della complessità ecologica?'. Nell'immensa disponibilità di sapere letterario/scientifico stimolata dal rigore di Ben in un contesto dove l'essere umano sembra ritrovare perdute connessioni naturali tra mente e corpo, emerge drammaticamente la mancanza due funzioni indispensabili: la cura e la socializzazione.

L'assenza trasversale della madre Leslie crea il necessario vuoto nel quale ricercare la risposta alle domande precedentemente formulate. La sua morte ingigantisce le lacune accettate da Ben, rigidamente convinto di proseguire il suo progetto comunitario nonostante le ormai evidenti, agli occhi dei figli, limitazioni di un sistema che non è riuscito a prendersi cura delle sofferenze materne e che impedirebbe alla famiglia di partecipare al funerale, quindi di condividere il lutto per la perdita dell'amata mogliemadre. L'entusiasmante scena che sancisce l'inizio dell' 'operazione di salvataggio' delle ultime volontà della madre è a conti fatti la prima decisione condivisa dalla famiglia di uscire dai confini dell'utopia.

Nell'ottica evolutiva del ciclo di vita, il percorso formativo coinvolge, infine, anche il padre. Ben scoprirà la propria impotenza in una scena in cui verrà definito 'fortunato', qualità che svela l'impossibilità di essere il totalizzante controllore della vita dei suoi figli. Guardando alla perduta onnipotenza, Ben sembra realizzare per la prima volta anche la sua 
condizione di vedovo; possono ora emergere i non detti di questa famiglia, adesso sollevata dal peso dell'utopia.

La decisione di rendere il film scorrevole grazie alle sue due ore, non permette d'altra parte di dare il giusto tempo alla complessizzazione della trasformazione familiare, peccato concesso e ampiamente giustificato dalla già ricca trattazione. Il momento dello strappo con il passato trova una, per certi versi forzata, sintesi che comunque ci lascia una pellicola che rigenera i nostri interrogativi, regalandoci una commedia piacevole e pensata.

Conflitto di interessi: l'autore dichiara che non vi sono potenziali conflitti di interessi.

Approvazione etica e consenso a partecipare: non necessario.

Ricevuto per la pubblicazione: 22 luglio 2020.

Accettato per la pubblicazione: 30 agosto 2020 .

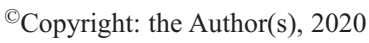

Licensee PAGEPress, Italy

Ricerca Psicoanalitica 2020; XXXI:312

doi:10.4081/rp.2020.312

This article is distributed under the terms of the Creative Commons Attribution Noncommercial License (by-nc 4.0) which permits any noncommercial use, distribution, and reproduction in any medium, provided the original author(s) and source are credited. 